\title{
A NEW LOOK AT FUNDAMENTALS OF THE PHOTOMETRIC LIGHT TRANSPORT AND SCATTERING THEORY. PART 1: ONE-DIMENSIONAL PURE SCATTERING PROBLEMS
}

\author{
S. Persheyev ${ }^{1}$ \\ D.A. Rogatkin ${ }^{2}$
}

sp95@st-andrews.ac.uk

\author{
${ }^{1}$ School of Physics and Astronomy, St Andrews University, St Andrews, \\ The United Kingdom of Great Britain and Northern Ireland \\ ${ }^{2}$ Moscow Regional Research and Clinical Institute named after M.F. Vladimirsky, \\ Moscow, Russian Federation
}

\begin{abstract}
In the majority of practical cases there exist difficulties with deriving an analytical closed-form solution of the classic radiative transport equation (RTE) in the light transport and scattering theory, which is widely used today in biomedical optics, ocean optics, atmospheric optics, etc. In our opinion, certain problems stem from the fact that the mathematical formulation of main physical processes at scattering in turbid media is not quite accurate. To study the problem in more detail, this paper once again describes and analyzes the photometric transport theory from the "first phenomenological principles". We show that this approach assists to clarify the problem in depth, as well as to obtain certain new, accurate and unexpected results. In this part 1 of the article, we consider in detail one-dimensional (1D) pure scattering problems featuring no absorption. We discuss and solve every main typical 1D pure scattering problem using various approaches. It allows us to prove that the scattering coefficient is not so much a real optical property of a turbid medium, but a parameter of the mathematical description of the problem. In the general case, the scattering coefficient depends on both optical properties of the medium and the mathematical approach selected. Therefore, it can vary with different approximations, which can be a source of errors in calculations
\end{abstract}

\section{Keywords}

Scattering, absorption, light transport, radiative transport equation, onedimensional problems, pure scattering, single scattering approximation, multiple scattering

Received 29.06.2017

(C) BMSTU, 2017

Introduction. The light transport and scattering theory (LT\&ST) is widely used today in biomedical optics, ocean optics, optics of atmosphere, etc. However, it is wellknown; there are difficulties with the analytical and closed-form solution of the classic radiative transport equation (RTE). In the general case, it does not have the analytical solution. Only a number of simplified approximations exist which have it. As we have reported in our previous publications, we think the problem follows from the not quite correct formulation of main equations in LT\&ST for different applications. In our opinion, for example, there is an error in description of the scattering 
coefficient as a native physical property of a light scattering (turbid) medium. In addition, some phenomenological nature of RTE and adopted universal form of RTE for all practical problems simplifies and distorts our perception of the complex mathematical formulation of the equation(s). Therefore, a number of other problems still remained in a shadow. These two statements we would like to prove from the "first principles" in the paper.

We will consider only stationary, time-independent problems, because they are exactly the fundamentals of the phenomenological LT\&ST. We work on biomedical optics, so examples for turbid biological tissues are more habitual to us. We will use them in this paper. However, our results are also applicable to other light scattering media clouds, milk glasses, turbid liquids, etc. We assume a reader is familiar with the classic LT\&ST. Therefore, there is no need to explain the basics of the theory, the basic items, and the existing variety of approaches to solve the direct task - Kubelka - Munk (KM) approximation, diffusion equation, Monte-Carlo simulation, etc., as well as to describe the difference between them. Nevertheless, we should make two preliminary notes.

1. Our note 1 concerns the photometric and phenomenological foundations of the theory. Fundamentals of the photometric LT\&ST were developed as early as the 18th century. That time no one understood in a proper manner the wave nature of light, especially its electromagnetic nature. LT\&ST was created by P. Bouger, J.H. Lambert, et al., as the pure ray theory - the theory describing light beam in terms of its power or energy as perceived brightness by human eyes. Neither diffraction nor interference were known that time in applications solving photometric issues. Understanding the waves, its phase, a power density as a square of the wave field came much later. In the original photometry, light beam was described as a radiant flux (beam of radiant rays with power) propagating and decreasing inside a continuous medium. The flux had an amplitude and a direction, but it was not a vector, because two fluxes were summed in the theory by amplitudes as scalars at intersection in a point of the medium, and not as vectors, unlike to electromagnetic fields. Classic RTE, which arose somewhat later, also, initially represented the pure photometric and phenomenological formulation of the problem.

The main law of the photometry is the exponential law discovered by P. Bouger in the beginning of 18th century, also known as Lambert - Bouguer law, Bouguer's law, etc. It relates the attenuation of the radiant flux $F$ to the absorption properties of the medium's substance, as well as to a distance, which the light travels inside the medium. Therefore, RTE is primarily based on the Bouguer's law. So, there is a sense to introduce it before the main issue. The simplest way to reproduce the Bouguer's law from the "first principles" is as follows. Assume that an external beam of light (flux) $F_{0}$ enters a material sample from the left side (Fig. 1). Determine the material sample is not light scattering, i. e., assume the pure absorption medium. Define $X$ as an axis parallel to the direction of the light beam propagation. Inside the sample, one can observe the radiant flux $F(x)$ of the light as a function of the fluent coordinate $x$. Select within the sample an element $\Delta x$ and define the decrement (increment) of the flux $F(x)$ inside $\Delta x$, obtaining the flux $F(x+\Delta x)$. 


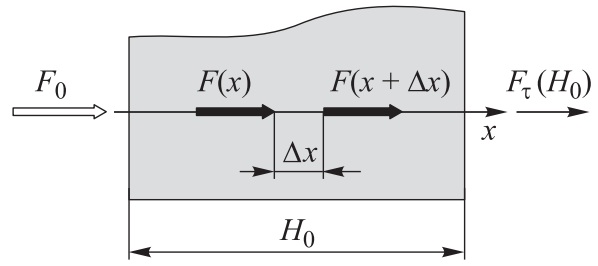

Fig. 1. Radiant flux $F(x)$ inside the continuous material sample and a formation of the flux decrement

The Bouguer's law initially was proved and confirmed in experiments, but we are interested in a theoretical derivation of the exponential law. For this purpose, one should assume the difference $\Delta F$ between $F(x+\Delta x)$ and $F(x)$. For example:

$$
\Delta F=F(x+\Delta x)-F(x)=-K F(x) \Delta x,
$$

where $K$ is the absorption coefficient. Its dimension is $[x]^{-1}$, i. e., the inverse dimension to a distance dimension. In the general case, $K$ is a function of wavelength $\lambda$, i. e. $K=K(\lambda)$. Hereinafter, for brevity and clarity, the dependence of the wavelength $\lambda$ are omitted. Equation (1) claims and defines the linear absorption of $F(x)$ inside $\Delta x$ with the coefficient of absorption $K=$ const. This coefficient can be not a constant, but a function of a coordinate $x$ for non-homogenous media. However, it is not a case for our consideration here. We consider just the simple case of the perfect homogenous, continuous medium, i. e. $K=$ const. According to the classic mathematical definition of the derivative

$$
\frac{d F(x)}{d x}=\lim _{\Delta x \rightarrow 0} \frac{\Delta F}{\Delta x}=-K F(x) .
$$

Therefore, ignoring boundary processes at $x=0$, one can obtain the following exponential law at $K=$ const:

$$
F(x)=F_{0} e^{-K x} .
$$

In the case of RTE, the more accepted notation for $K$ is $\mu_{a}$. As one can see, the main "first principle" in the Bouguer's law is the linear approximation in Eqs. (1) and (2). It tells us nothing about the inner structure as well as about the inner properties of the medium, with the exception of a uniformity of them along the $X$-axis. For example, if the substance of the sample is a medium with discrete absorbers (Fig. 2), each of which absorbs the part $a(a<1)$ of the radiant flux $F(x)$, and between absorbers there is no absorption at all, then

$$
\Delta F=F(x+\Delta x)-F(x)=F(x)(1-a)^{N}-F(x)=F(x)\left((1-a)^{N}-1\right),
$$

were $N$ is a number of absorbers " $a$ " inside $\Delta x$. Introducing the absorbers density $\mu_{\rho}$, once again with the dimension $[x]^{-1}$, substituting $N=\mu_{\rho} \Delta x$ into Eq. (4), and then Eq. (4) in Eq. (2), and revealing the infinity in Eq. (2) with the use of L'Hopital's rule, the limit in Eq. (2) can be evaluated as follows:

$$
\frac{d F(x)}{d x}=\lim _{\Delta x \rightarrow 0} \frac{\Delta F}{\Delta x}=-\left[-\mu_{\rho} \ln (1-a)\right] F(x) .
$$


Fig. 2. Radiant flux $F(x)$ and a formation of the flux decrement inside the sample with discrete absorbers

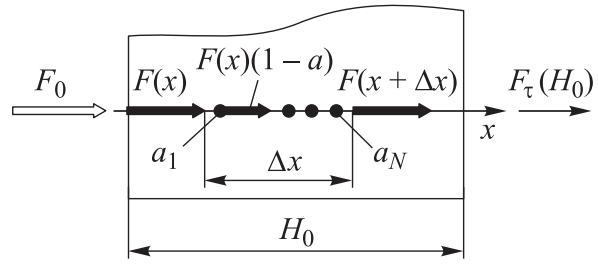

One can see, that defining $K=-\mu_{\rho} \ln (1-a)$, there is no way to distinguish between continuous and discrete absorbers media using both the photometric approach and measurements of $F(x)$. Despite the stepwise nature of the function Eq. (4), whose derivative, formally, does not exist at points of location of the absorbers, i.e., at points of discontinuity of the first kind, a smooth approximating curve for $F(x)$ can be found with the use of the formal phenomenological approach Eq. (2). Thus, there is no sense to consider models of discrete absorbers inside the material medium.

2. Our note 2 concerns the correspondence between photometry and electromagnetism. In spite of the lack of any needs in phenomenological photometry to understand the wave nature of light and the Maxwell's electrodynamics, nevertheless, it is important today to keep in mind the relationship between these two approaches. Mainly, it concerns the right understanding of the absorption and scattering properties of different turbid media in which light propagates. Many articles were published on this issue, but we need to subtract only the simplest statements what we are going to use for our next "first principles" approach. It consists of the following.

If consider a plane-parallel wave propagating along axis $X$ :

$$
E(x, t)=E_{0} e^{j\left(k x-\omega_{0} t\right)},
$$

where $E_{0}$ is the amplitude of the electric field $[\mathrm{V} / \mathrm{m}] ; j=\sqrt{-1}, k=2 \pi / \lambda$ is the wave number $\left[\mathrm{m}^{-1}\right] ; \lambda$ is the wavelength $[\mathrm{m}] ; \omega_{0}$ is the angular (circular) frequency; $t$ is time, then the radiant flux $F_{0}$ incident on the surface $A$ at a point $x$ is determined by the equation [1]

$$
F_{0}=\frac{A}{2 Z_{e}}|\overline{E(x, t)}|^{2},
$$

where $A$ is a surface $\left[\mathrm{m}^{2}\right] ; Z_{e}$ is the wave impedance of the medium $[\mathrm{Ohm}] ; \overline{E(x, t)}$ is the time-average field amplitude. It is well-known, that in a material medium the wave number $k$ can be expressed through the complex refractive index $n^{*}$ of the medium as follows:

$$
k=\frac{\omega_{0}}{c} n^{*}=\frac{\omega_{0}}{c}(n+j \chi)=\frac{2 \pi}{\lambda_{0}}(n+j \chi),
$$

where $c$ is the speed of light in vacuum; $n$ and $\chi$ are the real and imaginary parts of $n^{*}$ respectively; $\lambda_{0}$ is the wavelength in a free space. Imaginary part $\chi$ is proportional to 
the specific electrical conductivity of the medium, so the case of $\chi=0$ is the case of the perfect dielectric medium. Substitution Eq. (8) into Eq. (6) gives

$$
E(x, t)=E_{0} e^{-\frac{2 \pi \chi}{\lambda_{0}} x} e^{j\left(\frac{2 \pi n x}{\lambda_{0}}-\omega_{0} t\right)} .
$$

As we see, the real amplitude of the field is decreasing as the exponential function $\exp \left(-\frac{2 \pi \chi}{\lambda_{0}} x\right)$ inside all non-perfect dielectric media when $\chi \neq 0$. This result follows from fundamentals of the electromagnetic theory and has nothing to do with the phenomenology approach (Eqs. (1) and (2)). However, surprisingly, it gives the same result at $K=\frac{4 \pi \chi}{\lambda_{0}}=2 k_{0} \chi$, where $k_{0}$ is the wave number in a free space. Therefore, we have an accurate understanding of the nature of light absorption and its description in the phenomenological photometry. However, what about the scattering?

In basic Maxwell's electrodynamics, there is not such a special property of media as the scattering coefficient. Scattering of light as the process of dispersion of the radiation in different directions occurs with the appearance of inhomogeneities inside the medium and (or) of boundaries between media with different $n^{*}$. In the simplest case of a plane boundary, when a beam of light crosses the boundary line between two different media with different refractive index, reflection and refraction of the beam can be observed. Which fraction of the beam is reflected and which one is refracted (transmitted), as well as directions for both the reflection and refraction are described well by known Fresnel equations and Snell's law. For example, a fraction of the incident beam that is reflected from the plane boundary is given by the reflectivity $R(R \leq 1)$. At the normal incidence

$$
R=\frac{(n-1)^{2}+\chi^{2}}{(n+1)^{2}+\chi^{2}}
$$

In the common case, $R$ is a function of $\lambda$, i. e., $R=R(\lambda)$ due to both $n=n(\lambda)$ and $\chi=\chi(\lambda)$. Once again we see the connection with the electromagnetic properties of the medium, in this case through the complex refractive index $n^{*}$, but the conception of scattering is absent yet. It is absent in the basic diffraction theory, as well. Although, F. Grimaldi was first who carefully observed the effect of diffraction of light, and who also coined the term diffraction, from the Latin diffringere, exactly 'to break into pieces', referring to light breaking up into different directions, in electrodynamics this phenomenon is not described as scattering, so it does not need to involve the scattering coefficient. It just describes a light intensity as a function of "scattering" angle at light beam diffraction on an obstacle or on a slit when the dimension of them is roughly comparable to the wavelength. It can correspond to a phase function in LT\&ST, but not more. The item of scattering appears in electrodynamics at introduction of a rough boundary surface $[2,3]$ or at consideration of mutual optical 
heterogeneities inside the medium (Rayleigh scattering, Mie scattering, etc. [4]). In these cases, the diffraction technique is also used, but together with the statistical formalism. Scattering cross-section as an average value over particles and (or) over scattering angles is usually acted as the scattering properties of such a medium. However, this complex computational formalism has no clear expression by any simple formula. Moreover, the electromagnetic scattering cross-section is not identical to the photometric scattering coefficient. Often, for the turbid media of discrete particles the scattering coefficient is defined as an integral over the solid angle of the differential scattering cross-section for a single particle multiplied by average density of particles inside the medium $[5,6]$. Nevertheless, in our opinion, this diffraction-based approach with the use of diffraction integrals cannot be adopted as the "first principle" for the photometric LT\&ST. The scattering coefficient $\mu_{s}$, as it was initially involved in RTE, was the original turbid medium property, which was introduced purely heuristically, without the use of any items of electromagnetisms. Therefore, there is the need to study this problem in detail. Therefore, the issue of the scattering coefficient formation in the pure photometric theory will be our main objective for the first part of the article.

One dimensional scattering problems. Let us start with the simplest onedimensional (1D) scattering problem. Although, the 1D model seems to be very far from the reality, it is the basis of the Bouguer's law, of the Schuster - Schwartzchild approximation [7, 8], Kubelka - Munk (KM) approximation [5, 9], and of many other approaches, so the selection of such elementary model is not accidental, but is determined by a series of essential advantages. First, the simple 1D consideration of light ray absorption and scattering temporarily avoids complications with the definition of phase scattering functions, and therefore, allows us to concentrate on the phenomenological fundamentals of the scattering coefficient definition. Second, the $1 \mathrm{D}$ model is the simplest approximation of RTE. Having the precise and analytical solutions for all basic tasks, it opens a very powerful and convenient way to compare these solutions with any other results based on other approaches. Third, in full formulation with multiple scattering and absorption, the $1 \mathrm{D}$ model, being known more as the two-flux Kubelka - Munk (KM) model, has totally accepted opinion about its inaccuracy, as well as about disharmony of the results based on KM model and results based on RTE. Especially it concerns the compliance between scattering and absorption coefficients in KM approximation and in the general RTE. Thus, the study of the basic 1D scattering problems as fundamentals of LT\&ST is of great importance, in our opinion.

Preliminary remarks. Our experience on publications as well as conference presentations shows that professionals do not always understand 1D formulation of the scattering problem in the same way. Therefore, there is a need to clarify our $1 \mathrm{D}$ approach in the beginning of the section. In many publications, $2 \mathrm{D}$ or $3 \mathrm{D}$ radiative transfer problem is considered. Usually, the formulation of the problem looks like it is shown in Fig. 3 in the "flat" (plane layer) multidimensional formulation [10]. 


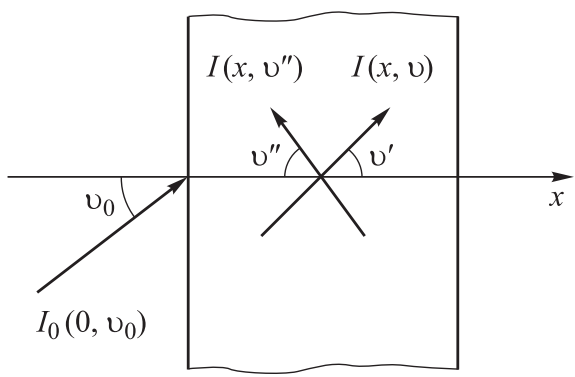

Fig. 3. Schematic "flat" representation of the radiation transfer in the multidimensional formulation

In the "flat" $2 \mathrm{D}$ representation, some radiance $I(x, \mathrm{v})\left[\mathrm{W} \cdot \mathrm{sr}^{-1} \cdot \mathrm{m}^{-2}\right]$, where $v$ is a polar angle $(I(x, v)$ also called as light intensity) propagates inside the turbid medium at the angle $v$ to the axis " $X$ ". For a narrow pencil of radiation, the radiance $I(x, v)$ and a radiant flux $F(x)$ are related by the equation $F(x)=I(x, v) \cos (v)[5,11]$. However, in the general 3D case, the flux $F(x)$ in a channel with a large solid angle $\Omega$ must be calculated from the radiance $I(x, v)$ by an integration over all angles included in the channel:

$$
F(x)=\frac{1}{2 \pi} \int I(x, v) \cos v d \Omega,
$$

where $\Omega$ is the solid angle. This approach was used, for example, by D. Yudovsky and L. Pilon [12]. Sometimes, using this approach, a "1D" RTE takes the form [13, 14]:

$$
\cos v \frac{d I(x, \mathrm{v})}{d x}=-\left(\mu_{a}+\mu_{s}\right) I(x, \mathrm{v})+\frac{\mu_{s}}{4 \pi} \int_{4 \pi} p\left(\mathrm{v}, \mathrm{v}^{\prime \prime}\right) I\left(x, \mathrm{v}^{\prime \prime}\right) d \Omega .
$$

where $\mu_{a}$ and $\mu_{s}$ are the absorption and scattering coefficients respectively, and $p\left(v, v^{\prime \prime}\right)$ is the scattering phase function. This approach is used since the introduction of the Schuster and Eddington approximations [8], and is very widespread due to the simplification of the coordinate system for RTE. However, this is not the pure 1D formulation of the problem. It is rather some 1D projection of a multidimensional task. It can be considered, but our objective is the pure 1D scattering problem, which does not contain any angles and directions of a radiance propagation different from the $X$-axis. We will consider the pure 1D turbid media, which is supposed to have only one line for the light transport - the axis " $X$ ", and there are not various angles at which light can travel.

Radiation penetrates the 1D turbid medium along the axis " $X$ ", and while propagating inside the medium is absorbed by the substance of the medium and (or) scattered by the heterogeneities of the inner structure of the medium. In the $1 \mathrm{D}$ problem, light rays are characterized by only two parameters: by directions of propagation (forward and backward), and by amplitudes, i.e. by a radiant flux with the dimension of watt $[\mathrm{W}]$. Radiant intensity $\left[\mathrm{W} \cdot \mathrm{sr}^{-1}\right]$ can be used as the amplitude characteristics of the light beam, as well, but it makes a little difference in the reasoning and mathematical formulation of all main equations in the $1 \mathrm{D}$ case. 
Our model of 1D turbid medium does not have a width, only a length $\left(H_{0}\right)$. Therefore, two fluxes only can exist inside the medium - a forward flux; we will denote it as $F_{+}(x)$, and a backward one; we will denote it as $F_{-}(x)$. In Figures, it is quite difficult to present a pure $1 \mathrm{D}$ scheme of light propagation, so for visibility to show propagating fluxes and some nuances of their scattering inside the medium we will illustrate the medium by a "thin" stick. An example of such a representation is shown in Fig. 4.

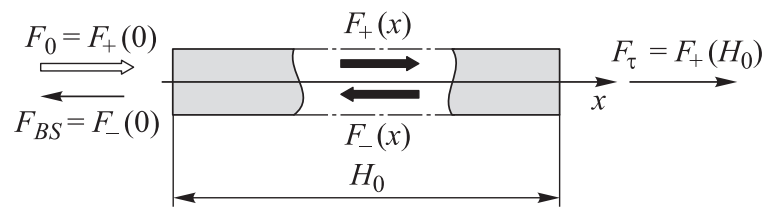

Fig. 4. Schematic representation of the $1 \mathrm{D}$ medium and propagating fluxes inside and outside it

The concept of diffraction of radiation in the 1D case has no any sense, because the diffraction is fundamentally a multidimensional phenomenon. In the 1D case, the scattering phase function is degenerated to the single opposite direction only, i. e. actually also does not exist (may not be taken into account). In the 1D problem, the only process that should be under consideration as scattering is the back-reflection of fluxes on "dotted" inhomogeneities along the $X$-axis. It is important to note here, that scattering as reflections is a discrete process on discrete inhomogeneities in principle. All it makes the problem to be effortless solved analytically in the closed form. The common approach used to solve the problem consists in formulation of differential equation for radiant fluxes changes at propagation inside a medium. We will use this technique, as well.

Pure scattering. As the first step, we will consider the case of the pure scattering. This is the limit case of the absence of absorption inside the turbid medium. From the electromagnetic point of view, it is the case of the perfect dielectric medium. Imaginary part of the refractive index inside the medium $\chi=0$. Therefore, $K=0$, so only scattering will take place. As we have mentioned above, we suspect there is an inaccuracy in the definition of the scattering coefficient in LT\&ST, and in incorporation of it into RTE. Therefore, the investigation on the scattering coefficient formation in a common phenomenological approach is more interesting for us. Pure scattering approximation give the best opportunity for the study. Thus, our task is to calculate a backscattered flux $F_{B S}=F_{-}(0)$, as well as a transmitted flux $F_{\tau}=F_{+}\left(H_{0}\right)$ as functions of optical properties of the medium. In the case of $1 \mathrm{D}$ pure scattering media, there are only a few physical properties of the media - a length $H_{0}$, a number $N$ of inhomogeneities distributed along the $X$-axis inside the medium, and the reflectivity $R_{i}$ for each $i$-th inhomogeneity.

Assume all $R_{i}=$ const $=R$. For a formation of the scattering coefficient item, it does not matter. Let all $N$ inhomogeneities $r_{i}$ be uniformly or some kind randomly distributed inside the medium. Figure 5 demonstrates the uniform distribution. 


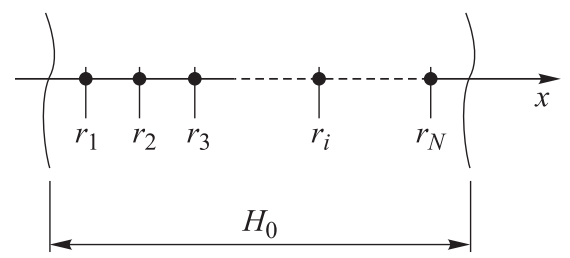

Fig. 5. Schematic representation of the $1 \mathrm{D}$ medium with $N$ inhomogeneities $r_{i}$ inside it

In next figures, we will not draw black dots to indicate inhomogeneities. We will simply denote their coordinates by short lines crossing the $X$-axis.

Single scattering approximation. The simplest case of the pure scattering model is the single scattering approximation (SSA). It gives the simplest solution of the $1 \mathrm{D}$ scattering problem. SSA assumes scattering (more exactly - the back reflection in the $1 \mathrm{D}$ case) for the forward flus $F_{+}(x)$ on each inhomogeneity (decrement of $F_{+}(x)$ after passing the inhomogeneity) and the absence of scattering for the backward flux $F_{-}(x)$, i. e. it assumes the negligible re-reflection process between any two heterogeneities inside the medium. Figure 6 explains the decrement of the flux $F_{+}(x)$ and a formation of fluxes $F_{-}(x), F_{B S}=F_{-}(0)$, and $F_{\tau}=F_{+}\left(H_{0}\right)$.
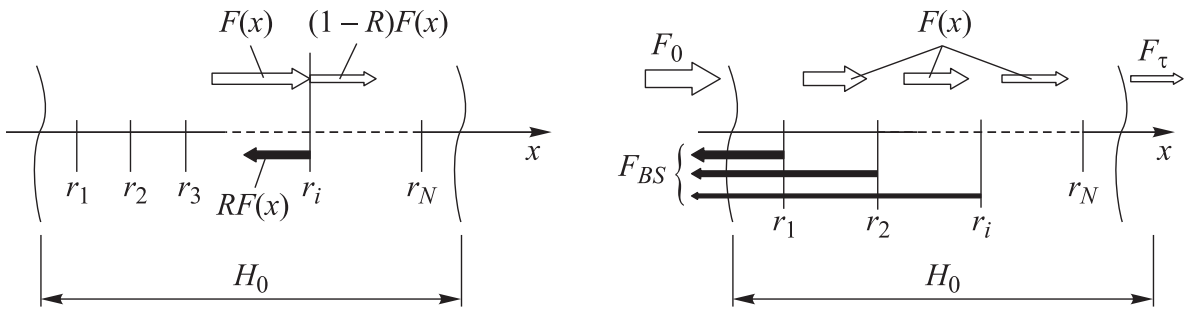

Fig. 6. Formation of the backscattered and transmitted fluxes in 1D SSA

It is evidently, that in this scheme:

$$
F_{\tau}=F_{+}\left(H_{0}\right)=F_{0}(1-R)^{N} .
$$

Therefore, for each elementary interval $\Delta x$, the decrement $\Delta F_{+}$of the propagating flux $F_{+}(x)$ will be equal:

$$
\Delta F_{+}=F_{+}(x+\Delta x)-F_{+}(x)=F_{+}(x)\left[(1-R)^{\mu_{\rho} \Delta x}-1\right],
$$

where $\mu_{\rho}\left[\mathrm{m}^{-1}\right]$ is the scatterers density inside $\Delta x$. One can see that Eq. (14) is mathematically identical to the Eq. (4), so, applying Eq. (5), it yields:

$$
\frac{d F_{+}(x)}{d x}=-\left(-\mu_{\rho} \ln (1-R)\right) F_{+}(x)=-S F_{+}(x),
$$

where by $S=-\mu_{\rho} \ln (1-R)$, following $\mathrm{KM}$ notations, we denoted the scattering

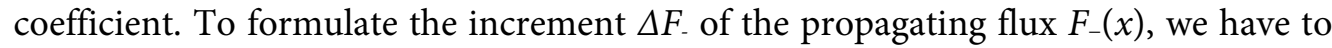
write [15]: 


$$
F_{-}(x)=F_{-}(x+\Delta x)+F_{+}(x) R\left[\sum_{i=1}^{N}(1-R)^{i-1}\right] .
$$

Note, there is the enhancement of $F_{-}(x)$ with the increment of $\Delta x$. The more size $\Delta x$ and $N$ are, the more $F_{-}(x)$ is formed due to the backscattering of $F_{+}(x)$. The sum in brackets is the geometric progression describing the decrement of $F_{+}(x)$ inside $\Delta x$. It can be calculated directly:

$$
\left[\sum_{i=1}^{N}(1-R)^{i-1}\right]=\frac{(1-R)^{N}-1}{(1-R)-1}=-\frac{(1-R)^{N}-1}{R} .
$$

Hence,

$$
\Delta F_{-}=F_{-}(x+\Delta x)-F_{-}(x)=F_{+}(x)\left[(1-R)^{N}-1\right]
$$

and finally:

$$
\frac{d F_{-}(x)}{d x}=-S F_{+}(x)
$$

that is similar to Eq. (15). Thus, we found a set of two coupled linear differential equations of the first-order describing the $1 \mathrm{D}$ pure scattering problem with the use of SSA:

$$
\begin{aligned}
& \frac{d F_{+}(x)}{d x}=-S F_{+}(x) ; \\
& \frac{d F_{-}(x)}{d x}=-S F_{+}(x) .
\end{aligned}
$$

At boundary condition $x=0: F_{+}(0)=F_{0} ; x=H_{0}: F_{-}\left(H_{0}\right)=0$, one can solve the system with the output:

$$
F_{+}(x)=F_{0} e^{-S x} ; \quad F_{-}(x)=F_{0}\left(e^{-S x}-e^{-S H_{0}}\right) .
$$

For professionals such a result is expectable and near trivial. Nevertheless, it is important to understand better all our next results. For example, we can note, that scattering exponential laws in Eqs. (21), similar to the Bouguer's law, is appeared here under the SSA as the direct consequence of SSA. Together with the obtained expression for the scattering coefficient at SSA

$$
S=-\mu_{\rho} \ln (1-R)
$$

it forms our first main output.

Multiple scattering approach. The case of multiple scattering is more interesting. For multiple scattering approach (MSA), we need to take into account all multiple reflections (re-reflections) between all scatterers $r_{i}$ inside the medium. Multiple scattering will lead to a phenomenon that every flux will be reduced in $\Delta x$ due to the reflections (scattering) on boundaries of inhomogeneities, as it was at single 
scattering; but, at the same time, it will be enhanced due to the backscattering of the opposite flux in $\Delta x$. Hence, the system of continuous differential equations for the multiple scattering case will has the form:

$$
\begin{aligned}
& \frac{d F_{+}(x)}{d x}=-S F_{+}(x)+S F_{-}(x) ; \\
& \frac{d F_{-}(x)}{d x}=S F_{-}(x)-S F_{+}(x) .
\end{aligned}
$$

Evidential solution for the system is

$$
\begin{aligned}
& F_{+}(x)=C_{1} x+C_{2} ; \\
& F_{-}(x)=C_{1} x+C_{3} .
\end{aligned}
$$

where $C_{2}$ and $C_{3}$ are constants, which should be determined using boundary conditions, and $C_{1}=S\left(C_{3}-C_{2}\right)$. At boundary conditions $x=0: F_{+}(0)=F_{0} ; x=H_{0}$ : $F_{-}\left(H_{0}\right)=0$, and we can find the fluxes as follows:

$$
F_{+}(x)=F_{0} \frac{1+S\left(H_{0}-x\right)}{1+S H_{0}} ; \quad F_{-}(x)=F_{0} \frac{S\left(H_{0}-x\right)}{1+S H_{0}} .
$$

It yields for the transmitted and backscattered fluxes respectively:

$$
F_{\tau}=F_{+}\left(H_{0}\right)=F_{0} \frac{1}{1+S H_{0}},
$$

and

$$
F_{B S}=F_{-}(0)=F_{0} \frac{S H_{0}}{1+S H_{0}} .
$$

We do not see the exponential law here! Fluxes at multiple pure scattering do not obey the exponential law. This is important and well-known result, but it is not all! Eqs. (25)-(27) tells us nothing about $S$. This is a key point. We can assume $S=-\mu_{\rho} \ln (1-R)$, like it was derived at SSA, but it will mean, evidently, that we assume SSA inside $\Delta x$ for each fluxes at MSA, and multiple scattering occurs due to mutual scattering between each $\Delta x$. It is the case $1-$ the quasi-multiple scattering process. It, probably, can be acceptable for a major number of turbid media, but here for the purpose of the investigation of theoretical basics of LT\&ST, we have to study the extreme case of multiple scattering directly inside $\Delta x$. This is the case $2-$ the perfect multiple scattering process. How can we do it? We know at least two ways. The more interesting way is based on the theory of Markov processes.

An assumption of the "photon" migration phenomenon in LT\&ST is now widely used in many applications, especially in statistical Monte-Carlo simulation. If this model is assumed, then the probability of the "photon" location at any point $x_{i}$ of the space can be interpreted like a probability of the state of the Markov $N$-section chain. In this case, the magnitudes of the parameter $t$ (time) in a function of conditional 
probability of transitions became quasi-discontinuous, and the possibility to replace them by the test number $n$ (or $m, l$, etc.) appears. In such approach the common probability of the "photon" transition $-p\left(n, x_{k} \mid l, x_{i}\right)-$ from $x_{i}$ point to $x_{k}$ point obeys the general Markov equation [16]:

$$
p\left(n, x_{k} \mid l, x_{i}\right)=\sum_{j} p\left(n, x_{k} \mid m, x_{j}\right) p\left(m, x_{j} \mid l, x_{i}\right), \quad l<m<n .
$$

For the homogeneous Markov chain all probabilities of the transitions using " $s$ " steps $(s=n-l)$ form a matrix of probabilities:

$$
p\left(x_{k} \mid s, x_{i}\right) \equiv \mathbf{p}_{i k}(s),
$$

herewith, if $N$ is a total number of chain's states which are taken into account, the sum of all matrix elements obeys the closing equation:

$$
\sum_{k=1}^{N} p_{i k}=1
$$

The equation (29) shows that probabilities of transitions with the use of any " $s$ " steps can be ultimately represented using only one-step probabilities. In fact, it reduces the solution of the Markov process problem to forming one-step matrixes and making the formal multiplications of them.

Because of $R \leq 1$ in our scattering model, reflectivity $R$ can be interpreted as a probability of the "photon" transition from one state to another when it is reflected by a heterogeneity $r_{i}$, whilst the probability of the opposite event for the "photon" (crossing the heterogeneity $r_{i}$ ) will be equal $1-R$. For the correct usage of the Markov processes mathematical formalism, it is necessary to enumerate all states of "photons". Consider $N$ heterogeneities $r_{i}$ inside $\Delta x$. If the simplest enumeration is chosen: before all $r_{i}$ (before $\Delta x$ ) there is the state number $i=1$, between $r_{1}$ and $r_{2}-$ the state number $i=2$, between $r_{2}$ and $r_{3}$ the state number $i=3$, etc., then one can determine all one-step probabilities for the "photon" migration. Excluding states $i=1$ and $i=N+1$ (after $r_{N}$ ), the transition from any $i$-th state to the state $i=i+1$ or $i=i-1$ has the probability $1-R$. The probability of staying in the state $i$ is $R$ (the case of backreflection in $i$-th state). If "photon" goes out of the $\Delta x(i=1$ or $i=N+1)$, the probability of next changing its state falls down to zero. But for this simplest enumeration approach, the Markovian properties of the process are violated: the transition from $i$-th state to $i+1$ or $i-1$ states depends on the prehistory of the "photon" traveling (was the "photon" moved from left to right or from right to left). Therefore, the problem cannot be resolved using the formalism of Markov processes.

To overcome this difficulty, we offered to consider the much more useful enumerating approach. The even numbers $(i=2,4,6, \ldots)$ we will use for the enumeration of the "photon" states between $r_{i}$ and out of them when "photon" travels from right to left. The uneven (odd) numbers $(i=1,3,5, \ldots)$ we will use when a 
"photon" travels from left to right. Thus, initial "photons" penetrating $\Delta x$ through the left boundary have the state $i=1$, backscattered from $\Delta x$ "photons" have the state $i=2$ and transmitted by $\Delta x-$ the state $i=2 N+1$. Strictly speaking, for this new enumerating scheme for every even state $i$, excluding $i=2$, only states $i-1$ or $i-2$ are permitted for a transition with probabilities $R$ and $1-R$ respectively. For every uneven state $i$, excluding $i=2 N+1$, the permitted states are $i+1$ and $i+2$. Any transitions from $i=2$ or $i=2 N+1$ states to any other states are not permitted (these transition probabilities equal to zero). After creating such a statistical scheme, one can start the general calculation.

To determine a backscattered radiation inside $\Delta x$ at multiple pure scattering, the total probability $P_{s}(N)$ of a "photon" transition from the state $i=1$ to the state $i=2$ through any $s$ steps $(s=1,2, \ldots, \infty)$ should be derived as a function of a number of heterogeneities $N$. It means that we must find the unlimited sum:

$$
P_{s}(N)=\sum_{s=1}^{\infty} p_{12}^{N}(s) .
$$

The matrix of all one-step transitions $\pi_{1}$, for example, in the case of $N=2$, is the matrix $\mathbf{p}_{i k}^{N=2}(s=1)$ with the dimension of $6 \times 6$ :

$$
\pi_{1}=\mathbf{p}_{i k}^{2}(1)=\left(\begin{array}{cccccc}
0 & R & 1-R & 0 & 0 & 0 \\
0 & 0 & 0 & 0 & 0 & 0 \\
0 & 0 & 0 & R & 1-R & 0 \\
0 & 1-R & R & 0 & 0 & 0 \\
0 & 0 & 0 & 0 & 0 & 0 \\
0 & 0 & 0 & 1-R & R & 0
\end{array}\right) .
$$

The probabilities of reaching the state $i=2$ from the state $i=1$ with the use of any $s$ steps can now be calculated by multiplication of corresponding matrixes (32). For example, if $N=2$, then the corresponding probabilities are:

$$
\begin{aligned}
& s=1: p_{12}(1)=R \\
& s=2: p_{12}(2)=0 \\
& s=3: p_{12}(3)=R(1-R)^{2} ; \\
& s=4: p_{12}(4)=0 \\
& s=5: p_{12}(5)=R^{3}(1-R)^{2} ; \\
& s=6: p_{12}(6)=0 ; \\
& s=7: p_{12}(7)=R^{5}(1-R)^{2}, \text { etc. }
\end{aligned}
$$

Thus, the sum of them is a series:

$$
P_{s}(2)=\sum_{s=1}^{\infty} p_{12}^{2}(s)=R+R(1-R)^{2}+R^{3}(1-R)^{2}+\ldots
$$


Starting from the second term of the series, the trivial infinitely decreasing geometrical progression is evident. Therefore, the limit for Eq. (33) is:

$$
P_{s}(2)=\frac{2 R}{1+R} .
$$

Repeating calculations for $N=3,4,5, \ldots$ It is easy to find the general probability $P_{s}(N)$ as the solution of our task for the backscattered radiation at MSA inside $\Delta x$. It has the form:

$$
P_{s}(N)=\frac{N R}{1+(N-1) R} .
$$

Substituting $\Delta x$ into the Eq. (27) instead of $H_{0}$, substituting $N=\mu_{\rho} \Delta x$, and equating (27) and (35), it is easy to derive the scattering coefficient definition at perfect MSA inside $\Delta x$ as follows:

$$
S=\frac{\mu_{\rho} R}{1-R} .
$$

The difference between Eq. (36) and Eq. (22) is noticeable. It is determined by the factor

$$
\gamma_{R}=\frac{-(1-R)}{R} \ln (1-R)
$$

which is presented in Fig. 7.

For a small magnitude of $R$, for example, for $R<0.05$, the difference is not significant, low than $10 \%$. Turbid biological tissues, for example, have a quite low conductivity (imaginary part of the refractive index) in the waveband of light. $R$ usually is not much than 0.1 , therefore the SSA can be acceptable. However, for biological liquids like blood, this difference has a considerable

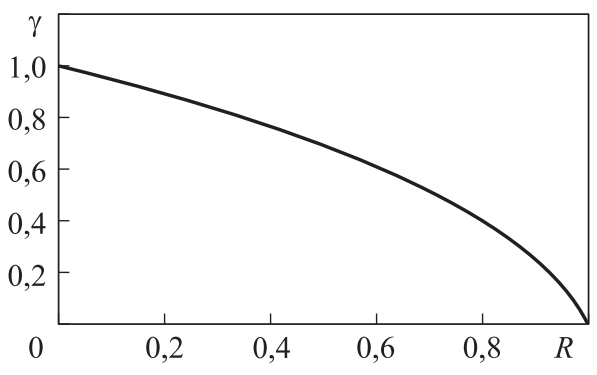

Fig. 7. Difference between scattering coefficients for perfect MSA and SSA impact on results, so the multiple scattering approach should be applied. It can be illustrated, for example, by ratios of backscattered fluxes and transmitted fluxes, given by Eqs. (26) and (27) respectively, if different $S$ given by Eq. (22) or Eq. (36) is used for computation. Both cases are cases of MSA due to the usage of Eqs. (26) and (27) as solutions, but the first one, with $S$ given by Eq. (22), is the case of multiple scattering in terms of the macro-medium, but with the single scattering inside $\Delta x$ (case 1), while the second one, with $S$ given by Eq. (36), is the pure multiple scattering outright with the multiple scattering inside $\Delta x$ (case 2). Figure 8 illustrates differences between these two cases. We have to note, that $H_{0}$ affects the result in the same way like $\mu_{\rho}$ because of their direct product in Eqs. (26) and (27). 

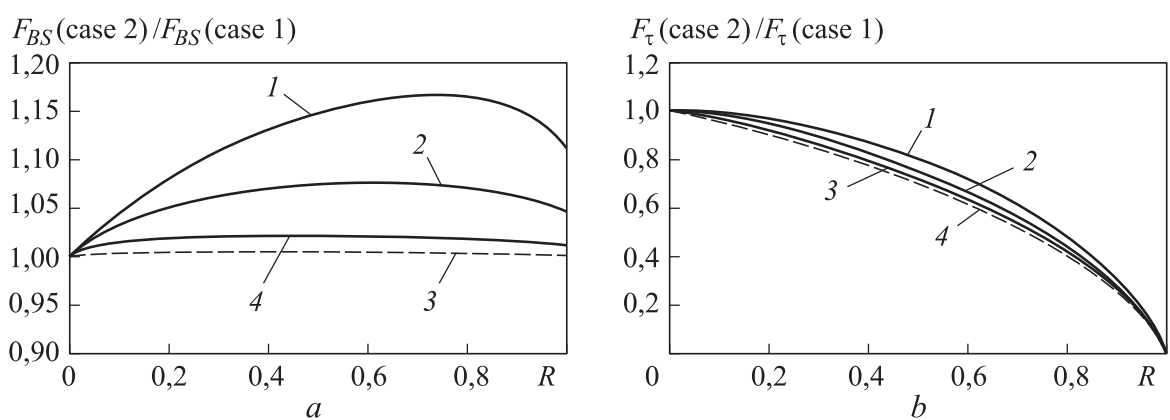

Fig. 8. Differences in backscattered $(a)$ and transmitted $(b)$ fluxes for two cases of multiple scattering model:

$$
1-\mu_{\rho} H_{0}=2 ; 2-\mu_{\rho} H_{0}=5 ; 3-\mu_{\rho} H_{0}=20 ; 4-\mu_{\rho} H_{0}=100
$$

Much stronger differences can be found if to compare solutions for MSA given by Eqs. (26), (27), (36) (case 2) with the similar solutions for SSA given by Eqs. (21), (22), i. e., with the use of both different scattering models and different scattering coefficients. These results are presented in Fig. 9.
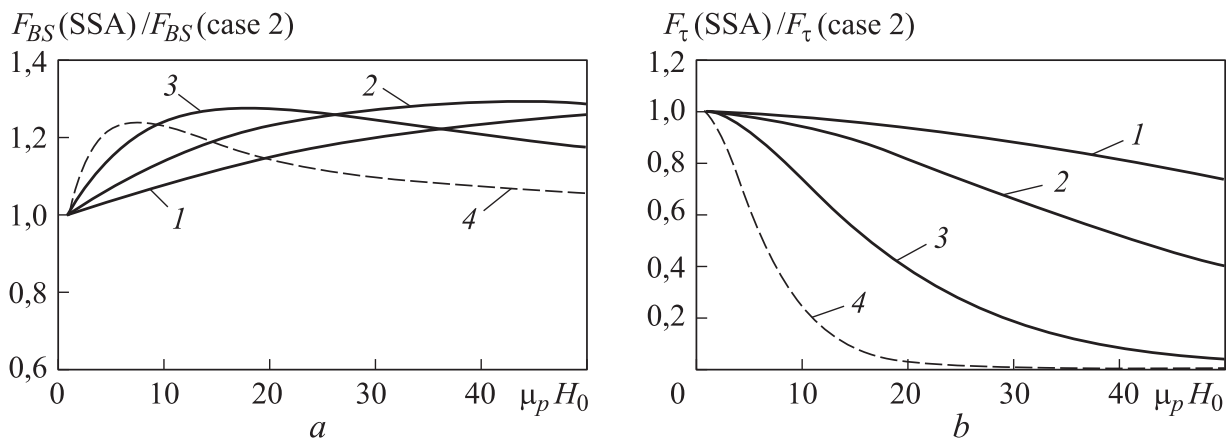

Fig. 9. Differences in backscattered ( $a$ ) and transmitted $(b)$ fluxes for SSA and the case 2 of MSA:

$$
1-R=0.02 ; 2-R=0.04 ; 3-R=0.1 ; 4-R=0.25
$$

As one can see, there are overestimated backscattered fluxes and, therefore, underestimated transmitted fluxes at SSA. The multiple scattering affects like a translucent factor at zero absorption.

Now, at the end of this section on pure scattering, as the first important conclusion we have to put the main question. We considered the same medium Fig. 5, the same problem of computing backscattered and transmitted fluxes, with the use of two different approaches: SSA and MSA. However, we obtained two different scattering coefficients Eqs. (22) and (36). Which one is correct, and which one is wrong? The medium cannot have two scattering coefficients at the same time. Therefore, only one answer is true: the scattering coefficient is a mathematical parameter of the approach used, of the approximation, not of the medium. The medium just has real physical parameters such as $R, H_{0}$ and $\mu_{\rho}$. Scattering coefficient 
$S$ (or $\mu_{s}$ ) is the parameter introduced by researchers to describe the scattering process as a continuous process using appropriate mathematical tools.

Conclusion. The light transport and scattering theory (LT\&ST) is widely used today in biomedical optics, ocean optics, optics of atmosphere, etc. However, there are difficulties with the analytical and closed-form solution of the classic radiative transport equation (RTE). In most practical cases, RTE does not have the analytical solution. As we have reported in our previous publications, the problem in particular follows from the not entirely accurate formulation of some main terms in LT\&ST. In this part of the article, we tried to prove one of these our assumptions from the "first principles", describing systematically a number of different models of 1D pure scattering problems. It was shown, that the scattering coefficient is a parameter of both optical properties of the medium and the mathematical approach used. It depends not only on optical properties of the medium, but also on the approximation, which is applied to solve the problem. Scattering coefficient in RTE is the photometric parameter, which was introduced to describe the scattering process as a continuous process using appropriate mathematical tools. Therefore, in different tasks it can vary, that can be a source of errors in calculations. Indeed, more real and close to realistic practical problems are problems of scattering with absorption. We will consider them in the second part of the article.

\section{REFERENCES}

[1] Stratton J.A. Electromagnetic theory. New York: McGraw-Hill, 1941. 573 p.

[2] Isakovich M.A. Scattering of waves from statistically rough surface. Zh. Éksperiment. Teor. Fiziki, 1952. vol. 23, no. 3, p. 305 (in Russ.).

[3] Beckmann A., Spizzichino P. The scattering of electromagnetic waves from rough surfaces. New York, Pergamon Press, 1963. 503 p.

[4] Newton R.G. Scattering theory of waves and particles. New York, McGraw-Hill, 1966. 699 p.

[5] Ishimaru A. Wave propagation and scattering in random media. New York, London, Academic Press, 1978. 272 p.

[6] Kokhanovsky A. Light scattering media optics. Problems and solutions. Chichester, Praxis Publishing Ltd., 2004. 320 p.

[7] Sobolev V.V. Perenos luchistoy energii v atmosferakh zvezd i planet [Radiative energy transfer in stellar and planetary atmosphere]. Moscow, Gosudarstvennoe izdatelstvo tekhnikoteoreticheskoy literatury Publ., 1956. 353 p.

[8] Sobolev V.V. Light scattering in planetary atmospheres. New York, Pergamon Press, 1975. $274 \mathrm{p}$.

[9] Kubelka P., Munk F. A contribution to the optics of pigments. Zeitung von Technologie und Physik, 1931, no. 12, pp. 593-599.

[10] Eliseev V.N., Tovstonog V.A. Teploobmen i teplovye ispytaniya materialov i konstruktsiy aerokosmicheskoy tekhniki pri radiatsionnom nagreve [Heat transfer and heat testing of materials and aerospace structures under radiant heating]. Moscow, Bauman MSTU Publ., 2014. $396 \mathrm{p}$. 
[11] Mudgett P.S., Richards L.W. Multiple scattering calculations for technology. Appl. Opt., 1971, vol. 10, iss. 7, pp. 1485-1502. DOI: 10.1364/AO.10.001485

[12] Yudovsky D., Pilon L. Simple and accurate expressions for diffuse reflectance of semiinfinite and two-layer absorbing and scattering media. Appl. Opt., 2009, vol. 48, iss. 35, pp. 6670-6683. DOI: 10.1364/AO.48.006670

[13] Handapangoda C.C., Pathirana P.N., Premaratne M. Eigen decomposition solution to the one-dimensional time-dependent photon transport equation. Optics Express, 2011, vol. 19, iss. 4, pp. 2922-2927. DOI: 10.1364/OE.19.002922

[14] Selden A.C. Accurate radiative transfer calculations for layered media. J. Opt. Soc. of Am. A, 2016, vol. 33, iss. 7, pp. 1409-1414. DOI: 10.1364/JOSAA.33.001409

[15] Dmitriev M.A., Fedukova M.V., Rogatkin D.A. On one simple backscattering task of the general light scattering theory. Proc. SPIE, 2004, vol. 5475, pp. 115-122.

DOI: $10.1117 / 12.568557$

Available at: https://spie.org/Publications/Proceedings/Paper/10.1117/12.568557

[16] Rytov S.M. Vvedenie v statisticheskuyu radiofiziku. Chast 1. [Introduction to the statistical radiophysics. Vol. 1]. Moscow, Nauka Publ., 1976. 491 p.

Persheyev S. - Dr. Sc. (Eng.), Research Fellow, School Physics and Astronomy, St Andrews University (College Gate, St Andrews KY16 9AJ, Fife, Scotland, UK).

Rogatkin D.A. - Dr. Sc. (Eng.), Assoc. Professor, Head of Medical and Physical Research Laboratory, Moscow Regional Research and Clinical Institute named after M.F. Vladimirsky (Shepkina ul. 61/2, Moscow, 129110 Russian Federation).

\section{Please cite this article in English as:}

Persheyev S., Rogatkin D.A. A New Look at Fundamentals of the Photometric Light Transport and Scattering Theory. Part 1: One-Dimensional Pure Scattering Problems. Vestn. Mosk. Gos. Tekh. Univ. im. N.E. Baumana, Estestv. Nauki [Herald of the Bauman Moscow State Tech. Univ., Nat. Sci.], 2017, no. 5, pp. 78-94. DOI: 10.18698/1812-3368-2017-5-78-94 